\title{
Emerging techniques and efficacy of endoscopic esophageal reconstruction and lumen restoration for complete esophageal obstruction
}

Authors

Institutions
Yaseen Perbtani ${ }^{1, *}$, Alejandro L. Suarez ${ }^{2, *}$, Mihir S. Wagh ${ }^{3, *}$

${ }^{1}$ Department of Medicine University of Florida, Gainesville, Florida, United States

${ }^{2}$ Division of Gastroenterology, Medical University of South Carolina, Charleston, South Carolina, United States

${ }^{3}$ Division of Gastroenterology, University of Florida, Gainesville, Florida, United States

\section{Bibliography}

Dol http://dx.doi.org/

10.1055/s-0041-107898

Published online: 11.1.2016

Endoscopy International Open 2016; 04: E136-E142

(c) Georg Thieme Verlag KG

Stuttgart · New York

E-ISSN 2196-9736

\section{Corresponding author}

Mihir S. Wagh, MD, FACG,

FASGE.

Interventional Endoscopy

Division of Gastroenterology

University of Colorado

1635 Aurora Court, F735

Aurora, CO 80045

USA

Phone: $1+720-848-2786$

Fax: 1+720-848-2749

mihir.wagh@ucdenver.edu
Background and study aims: Complete esophageal obstruction (CEO) is a rare occurrence characterized by progressive esophageal stricture, which eventually causes lumen obliteration. With recent advances in flexible endoscopy, various innovative techniques exist for restoring luminal continuity.

The primary aim of this study was to assess the efficacy and safety of patients undergoing combined antegrade-retrograde endoscopic dilation for CEO at our institution. The secondary aim was to review and highlight emerging techniques, outcomes, and adverse events after endoscopic treatment of CEO.

Patients and methods: Our electronic endoscopy database was retrospectively reviewed to identify patients who underwent combined antegrade and retrograde endoscopy for CEO. Patient and procedural data collected included gender, age,

\section{Introduction}

\section{$\nabla$}

Complete esophageal obstruction (CEO) is an uncommon phenomenon characterized by lumen obliteration that can arise from both benign and malignant etiologies. The former, being more frequent, is commonly the result of chemoradiation therapy of head and neck and lung cancers. However, incidence is dose-dependent with occurrence stated to be between $0.8 \%$ to $5 \%$ in those who have over $60 \mathrm{~Gy}$ exposure [1]. The underlying pathophysiology is likely progressive chronic inflammatory changes with subsequent fibrosis and collagen deposition from radiation or toxic exposures. A connective tissue membrane obliterating the esophageal lumen frequently is encountered as a result of this process. Longer-seg-

* Contributorship: data collection and analysis, draft and final approval of manuscript, Y.P.; data analysis, draft and final approval of manuscript, A.S.; concept and draft analysis, draft and final approval of manuscript, M.W. technical success, pre- and post-dysphagia scores, and adverse events.

Results: Six patients (67\% male, mean age 71.6 years [range 63-80]) underwent technically successful esophageal reconstruction with combined antegrade-retrograde endoscopy. All patients noted improvement in dysphagia with mean preprocedure dysphagia score of 4 reduced to 1.33 (range $0-3$ ) post-procedure. There were no adverse events and mean follow-up time was 17.3 months (range 3-48).

Conclusions: Combined antegrade and retrograde endoscopic therapy for CEO is feasible and safe. We present our experience with endoscopic management of complete esophageal obstruction, and highlight emerging techniques, outcomes and adverse events related to this minimally invasive modality.

ment completely obstructing fibrotic strictures also can occur, making endoscopic lumen restoration challenging. Typical clinical symptoms include severe dysphagia or aphagia with the inability to tolerate secretions. Esophagram and direct visualization of the obstructed "blind"esophageal lumen allows for diagnosis. Blind antegrade endoscopic puncture or dilation is usually not performed due to risk of injury to surrounding critical structures in the neck and chest. Hernandez et al [2] demonstrated that blind antegrade dilation can in fact lead to higher rates of complications such as perforations and fistula formation in complex strictures. Treatment options have been technically challenging with some including radical surgical procedures such as esophagectomy with colonic interposition, gastric transposition or platysma myocutaneous flap repair, all of which have substantial morbidity and mortality [3].

Consequently, minimally invasive approaches using endoscopy to restore luminal patency have 


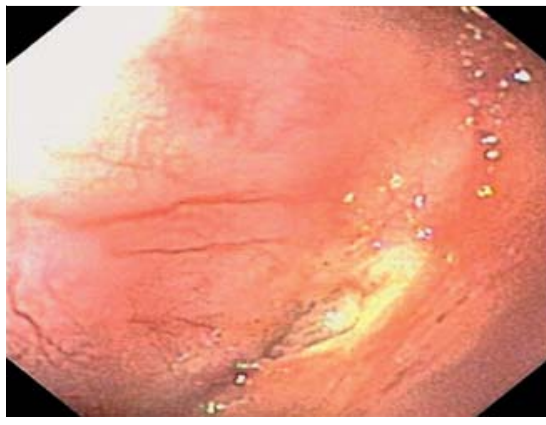

Fig. 1 Endoscopic view showing complete esophageal obstruction ("blind esophagus").

been the mainstay in recent years. In a sentinel paper Tucker [4] described management of complex esophageal strictures with a retrograde dilation approach through a mature gastrostomy site. Van Twisk et al [5] described a similar approach in which a flexible endoscope was inserted retrograde via a gastrostomy site for CEO. Currently, various other endoscopic methods are available for lumen restoration in CEO, including a combined antegraderetrograde endoscopic dilation or rendezvous procedure [6]. This approach became more commonly employed after a case series published by Bueno and colleagues [7]. The main limitation to this strategy is that it is restricted to esophageal obstructions typically less than $3 \mathrm{~cm}$ in length. However, we recently described a novel approach (POETRE: Per-Oral Endoscopic Tunneling for Restoration of the Esophagus) using endoscopic submucosal tunneling with combined antegrade-retrograde endoscopic dilation for longer segments of obstructed esophagus [8].

To date, results in more than 180 patients have been described in various published case series using different techniques and devices demonstrating excellent clinical symptom resolution with low rates of adverse events. In this article, we present our experience with endoscopic management of complete esophageal obstruction. In addition, we highlight emerging techniques, outcomes and adverse events related to this minimally invasive modality.

\section{Patients and methods}

$\nabla$

This study was approved by the University of Florida Institutional Review Board (IRB). Our electronic endoscopy database was queried from January 2009 through June 2014 for patients who were referred for antegrade and retrograde endoscopy for CEO. Diagnosis of the CEO was made with clinical history and direct endoscopic visualization. General anesthesia was used per anesthesiologist recommendations and prophylactic antibiotics were typically not given unless a submucosal tunneling technique was undertaken

The primary aim of the study was to assess the efficacy and safety of combined antegrade-retrograde endoscopic dilation for CEO in patients undergoing the procedure at our institution. The secondary aim was to review and highlight emerging techniques, outcomes, and adverse events (AEs) after endoscopic treatment of CEO.

Efficacy was defined by: (1) technical success of endoscopic therapy; and (2) clinical success as determined by improvement in dysphagia score. Safety was assessed by monitoring intra- and post-procedural AEs.

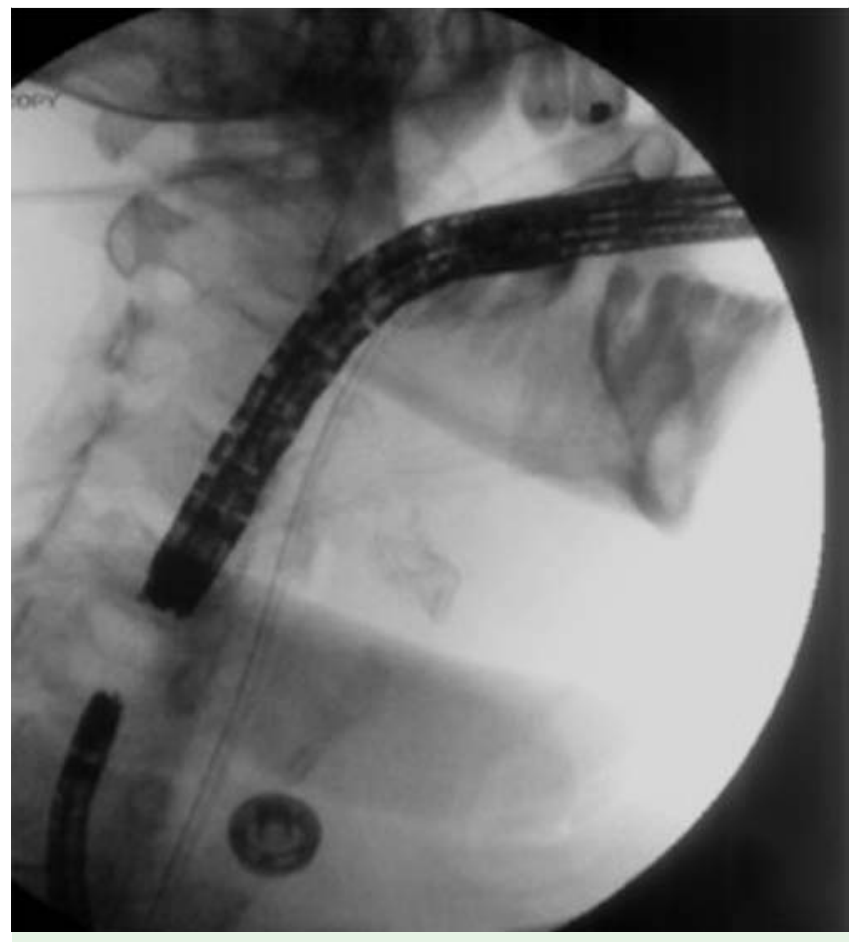

Fig. 2 Fluoroscopic view of antegrade and retrograde endoscopes approaching each other during combined endoscopy.

\section{Definitions}

Technical success: Procedural technical success was defined as the ability to successfully perform simultaneous antegrade and retrograde endoscopy with dilation and restoration of esophageal continuity.

Dysphagia score: Score range $0-4.0=$ no dysphagia; 1 =dysphagia to solids; 2 =dysphagia to semisolids; 3 =dysphagia to liquids; 4 = patient unable to swallow saliva (complete dysphagia/aphagia) was used to quantify dysphagia prior to and after endoscopic treatment [9].

Adverse events: Endoscopic AEs were assessed based on criteria previously established by the American Society of Gastrointestinal Endoscopy (ASGE) [10].

\section{Procedural technique}

The procedure was begun by using an antegrade endoscope to demonstrate a CEO at or distal to the esophageal inlet ( Fig.1). A retrograde endoscope was then inserted through a mature gastrostomy site and guided up the esophagus. The proximity of the two endoscopes was confirmed by use of multiplanar fluoroscopy ( $\bullet$ Fig. 2 ) and transillumination from the endoscope ( $\bullet$ Fig.3). In cases where the distance between the two endoscopes was measured to be less than $3 \mathrm{~cm}$, a 19-gauge endoscopic ultrasound needle (EUS-N) was used to puncture and traverse the obstruction under multiplanar fluoroscopy and simultaneous antegrade and retrograde endoscopic guidance. This allowed guide wire access to the distal esophagus, which permitted the tract to be balloon dilated. When antegrade passage of the guidewire was not successful, the guide wire was advanced through the retrograde endoscope and probed with antegrade endoscopic guidance under fluoroscopy. The guidewire was seen to exit into the pharynx on antegrade views where the wire was then grasped with a snare through the antegrade endoscope. Balloon dilation was 


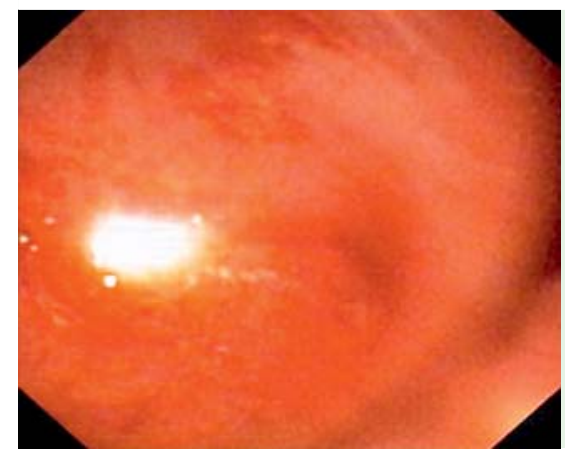

Fig. 3 Transillumination from the antegrade endoscope

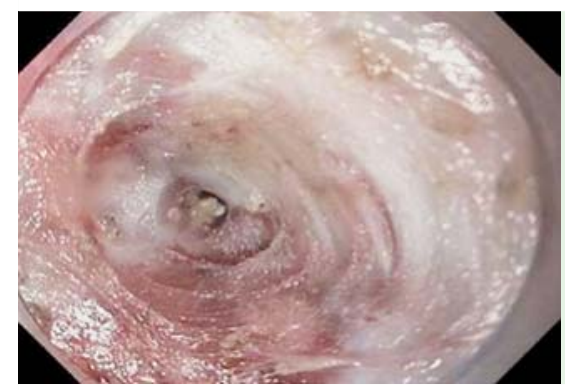

Fig. 4 Submucosal tunnel

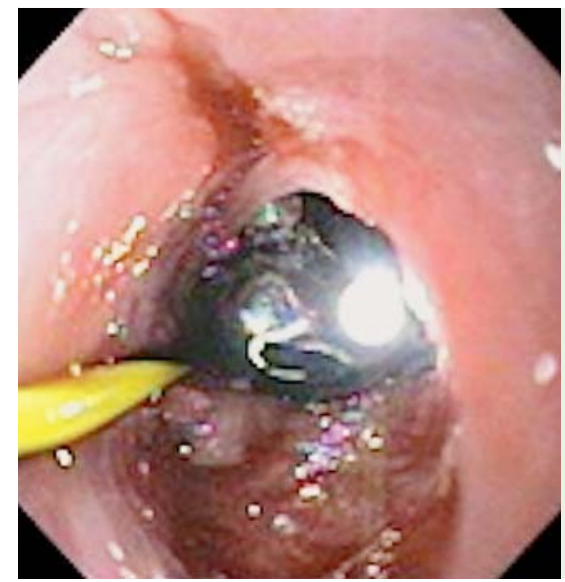

Fig. 5 Retrograde endoscope visible on antegrade views after reconnecting the esophagus. then performed under fluoroscopic guidance via the antegrade endoscope.

In cases in which simultaneous antegrade and retrograde endoscopes were visualized to be separated by a distance longer than $3 \mathrm{~cm}$, per-oral endoscopic tunneling (POETRE) [8] was done for restoration of the esophagus. A submucosal "bleb" was created with injection of saline-indigo carmine and submucosal tunneling ( Fig.4), which allowed the submucosal space to be entered with the anterograde endoscope. Submucosal tunneling then proceeded caudally and was achieved with repeat injections and dissection with a T-type Hybrid Knife (ERBE). When close proxi- mity of the two endoscopes was seen under fluoroscopy along with indentation from the approaching endoscope, the retrograde endoscope ( $\bullet$ Fig.5) was advanced into the proximal esophagus, thereby creating a neo-esophageal lumen and restoring esophageal continuity. A guidewire was passed through the retrograde endoscope, caught by a snare from the anterograde endoscope and pulled through the oral cavity. Finally, fully covered stents were deployed over the guidewire, which spanned the entire submucosal tunnel. The stents were removed in 4 to 6 weeks and serial endoscopic dilations were performed as needed to maintain esophageal patency ( $\bullet$ Fig. 6 a and $\bullet$ Fig. 6 b).

\section{Results \\ $\nabla$}

A total of 6 patients ( $67 \%$ male, $33 \%$ female, mean age 71.6 years [range 63-80]) with CEO were treated with flexible endoscopic therapy. Patient characteristics are summarized in $\bullet$ Table 1. Three patients had laryngeal cancer, 2 had pharyngeal cancer and 1 patient had a previous history of lung cancer. All of the patients had a history of chemoradiation therapy for their malignancy and also had G-tube placement prior to referral to our center. Four patients underwent technically successful rendezvous procedures, with the average size of obstruction being $\leq 3 \mathrm{~cm}$. Two patients, whose obstructions measured $4 \mathrm{~cm}$ and $5 \mathrm{~cm}$, respectively, underwent technically successful POETRE. One endoscopic treatment session was performed per patient and all patients noted improvement in their dysphagia symptoms after therapy. The mean pre-procedure dysphagia score was 4 and fell to 1.33 (range $0-3$ ) post-procedure. All patients required repeat dilations to remain luminal patency (mean 6.8 dilations [range 4-15]). There were no AEs and mean follow-up time was 17.3 months (range $3-48$ ). Two of the 6 patients no longer required G-tube feedings.

\section{Discussion \\ $\nabla$}

Currently, 14 case series/analyses [5,7,11-22] ( Table 2) consisting of 184 patients who underwent endoscopic treatment for CEO have been published. Radiation-induced strictures were thought to be the etiology in the majority (94.6\%) of the cases ( Table 3). Technical success was seen in 174/184 (95\%), of which 172/184 (93\%) were rendezvous procedures. Among the cases that were termed unsuccessful $[12,16,17]$, longer length of the obstruction was stated to be the sole cause.

In terms of outcomes, considerable heterogeneity exists in defining clinical success given the subjective nature of reported results and retrospective analysis. In studies where dysphagia scores

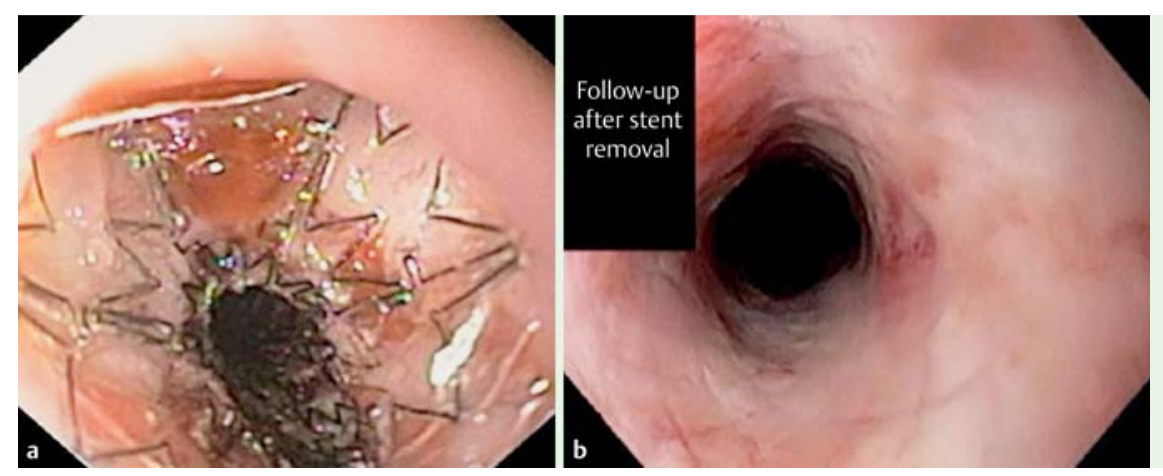

Fig. 6 a Fully covered stent across neo-esophageal lumen. b Esophageal stent removal showing luminal patency 
Table 1 Characteristics and outcomes of patients undergoing combined antegrade-retrograde endoscopy for complete esophageal obstruction.

\begin{tabular}{|c|c|c|c|c|c|c|c|}
\hline Patients & Age & Sex & Procedure & $\begin{array}{l}\text { Length of } \\
\text { obstruction }\end{array}$ & $\begin{array}{l}\text { Pre-dysphagia } \\
\text { score }\end{array}$ & $\begin{array}{l}\text { Post-dysphagia } \\
\text { Score }\end{array}$ & $\begin{array}{l}\mathrm{F} / \mathrm{U} \\
\text { (months) }\end{array}$ \\
\hline 1 & 65 & M & $\begin{array}{l}\text { Combined antegrade-retrograde } \\
\text { endoscopic dilation/rendezvous }\end{array}$ & $<3 \mathrm{~cm}$ & 4 & 0 & 14 \\
\hline 2 & 78 & $\mathrm{~F}$ & $\begin{array}{l}\text { Combined antegrade-retrograde } \\
\text { endoscopic dilation/rendezvous }\end{array}$ & $<3 \mathrm{~cm}$ & 4 & 1 & 48 \\
\hline 3 & 68 & M & $\begin{array}{l}\text { Combined antegrade-retrograde } \\
\text { endoscopic dilation/rendezvous }\end{array}$ & $<3 \mathrm{~cm}$ & 4 & 0 & 3 \\
\hline 4 & 63 & M & $\begin{array}{l}\text { Combined antegrade-retrograde } \\
\text { endoscopic dilation/rendezvous }\end{array}$ & $<3 \mathrm{~cm}$ & 4 & 2 & 15 \\
\hline 5 & 76 & $\mathrm{~F}$ & POETRE & $4 \mathrm{~cm}$ & 4 & 2 & 20 \\
\hline 6 & 80 & M & POETRE & $5 \mathrm{~cm}$ & 4 & 3 & 4 \\
\hline
\end{tabular}

were used, or interpretable from the collected data, the average pretreatment and posttreatment dysphagia score was 4 and 2.26 , respectively. The other authors based clinical success on symptom improvement post-procedurally. In studies where clinical success was reported, 113/139 (81\%) patients showed symptomatic improvement.

AEs from endoscopic therapy of CEO have been well documented since first being described. However, given the lack of standardization, there remain differences in how complications are reported and designated. Of the 184 reported patients who underwent endoscopic therapy for CEO, 36 (19.5\%) were reported to have an AE ( Table 4), the most common being micro-perforation, which occurred in $36 \%$ (13/36) of the cases. These were described as development of either subcutaneous or mediastinal emphysema. Most of these AEs were documented by various radiographic studies and the patients had inconsequential clinical outcomes and were treated conservatively without antibiotics. The next most common AE was malfunction of the G-tube which included loss of G-tube site, tract and subsequent leak following the procedure. All of these AES were likely to have occurred due to manipulation of the G-tube site during the procedure and were easily and successfully revised. Abscesses were the most common presentation of infections reported in published cases. Patient mortality was infrequent, occurring in only one case, reportedly [19, 23] due to a venous air embolism.

With recent advances in flexible endoscopy, several methods have been described for treating CEO, including antegrade, retrograde, and combined antegrade-retrograde endoscopic dilation with technical variations. In this article, we describe our experience along with other reported techniques and outcomes. As such, to date, good outcomes have been described in 190 patients, including those in our series. Because of the retrospective nature of the cases reported, some heterogeneity exists in preoperative and intraoperative techniques and postoperative care of these patients.

Typically, both barium esophagram and diagnostic endoscopy were done prior to any intervention. These tests help to identify the presence, level, and extent of the esophageal obstruction as factors such as location and length have been shown to be predictors of technical success [17]. In the largest single-center study of CEO reported, Goguen et al demonstrated that failure was most likely in cases involving larger obstructions and stenosis located in the region of the larynx or pharynx. In addition, if patients were to be considered for retrograde or combined antegraderetrograde endoscopic dilation therapy, a mature gastrostomy tract would be preferred, as manipulation of immature tracts in individuals who are malnourished or immunocompromised reportedly leads to higher rates of peritonitis [24].

Mode of sedation was dependent on patient and procedural characteristics. One advantage of flexible endoscopic therapy for CEO is the ability to perform the procedure without general anesthesia, thus allowing patients who are not optimal candidates for endotracheal intubation to undergo both moderate sedation (conscious sedation/CS) or deep sedation/monitored anesthesia care (MAC). These modes of anesthesia have been reported to be safe except when submucosal tunneling was performed $[12,15,16$, 22 ] and general anesthesia was used. Nevertheless, general anesthesia is still the most frequently reported form that has been used ( $\bullet$ Table 2 ).

Several methods described for endoscopic lumen restoration in CEO have been shown to be both effective and safe including antegrade, retrograde, combined antegrade-retrograde endoscopic dilation and the most recently described submucosal tunneling techniques. Single endoscopic antegrade dilations are however, difficult to achieve due to a frequently encountered fibrous membrane or longer segment of complete obstruction obliterating the view of the esophageal lumen. Any attempt at puncture for traversing the obstruction may lead to perforation or inadvertent injury to surrounding critical structures in the neck and chest. Nonetheless a novel approach was recently described using a EUS-guided puncture [25]. In this technique, the echoendoscope is placed on the proximal end of the obstruction. Visualization of the distal end of the CEO was obtained through EUS images. In conjunction with fluoroscopy, an EUS needle was used to puncture the obstruction, guidewire was passed through the needle, and subsequent dilations were performed to restore luminal patency. This technique is particularly useful in patients without a preexisting gastrostomy tract.

A retrograde approach to esophageal dilation, in which a swallowed string was used as a guide for bougie dilation, first was described in 1924 [4]. Since then, technical approaches to this method for CEO have typically described introduction through a gastrostomy tube site of a thin-diameter endoscope. This was followed by intubation of the esophagus with the endoscope and advancement of a guidewire to traverse the obstruction and feed it into the oral cavity. Subsequently, dilators were passed over the guidewire for serial dilations. In cases in which the guidewire could not be passed, successive retrograde balloon dilations have been described [15].

Combined antegrade-retrograde endoscopic dilation for CEO utilizes a collaborative approach between two endoscopists to meet at both the superior and inferior edges of the obstruction. Earlier reports described the procedure using a rigid esophagoscope via 


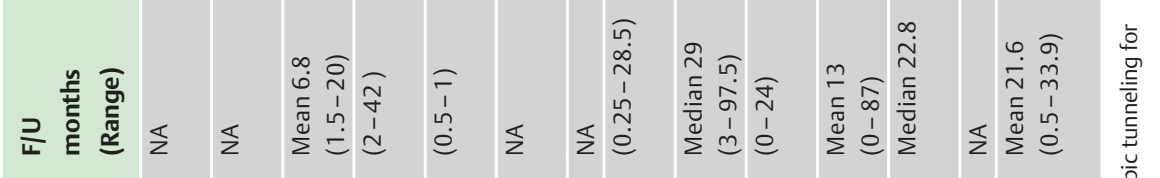
㞸

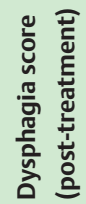

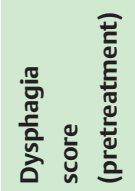

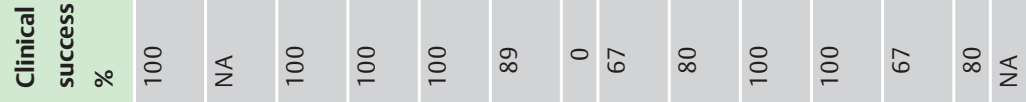

厄ֶ气

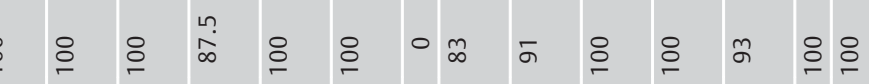

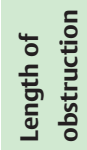

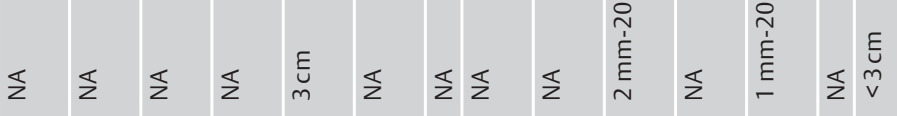

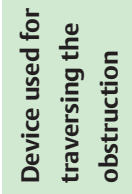

高

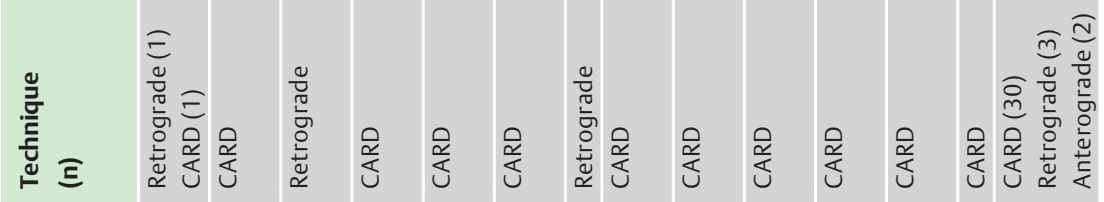

즘

言

帝

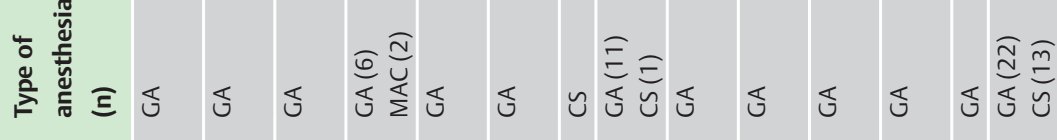

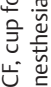

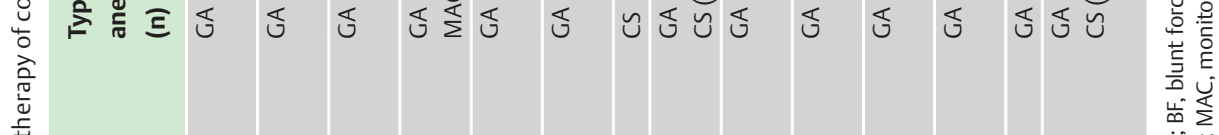

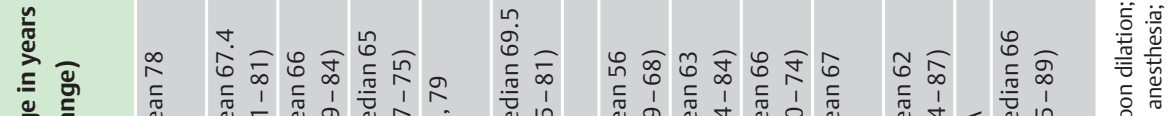

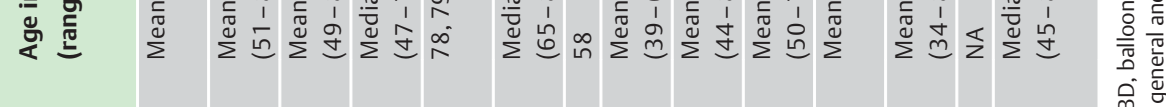

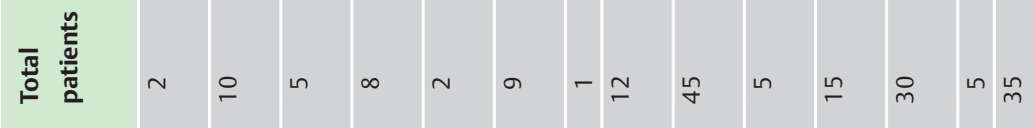

梂芯

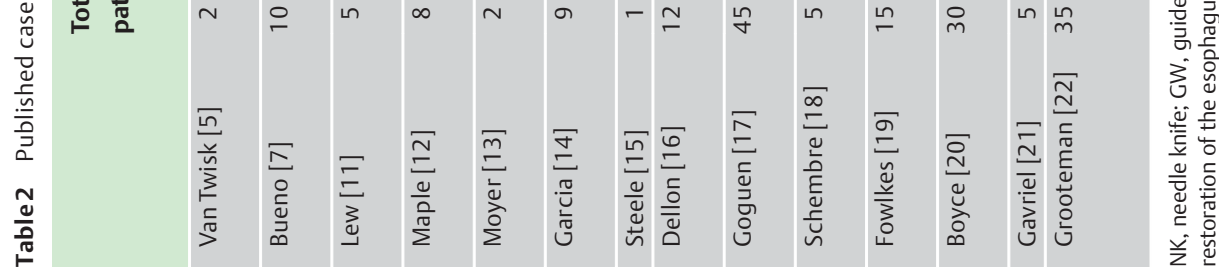


Table 3 Causes reported for complete esophageal obstruction

\begin{tabular}{|l|c|}
\hline Obstruction type & $\begin{array}{l}\text { Patients } \\
\text { (n=184) }\end{array}$ \\
\hline Radiation, $\mathrm{n}(\%)[5,7,11-22]$ & $174(94.6)$ \\
\hline Malignancy, $\mathrm{n}(\%)[7,11,14]$ & $4(2.2)$ \\
\hline Postsurgical anastomosis, $\mathrm{n}(\%)[7,14]$ & $5(2.7)$ \\
\hline Cricopharyngeal hypertrophy, $\mathrm{n}(\%)[5]$ & $1(0.5)$ \\
\hline
\end{tabular}

the mouth and a flexible endoscope via the G-tube tract. Flexible endoscopes currently are preferred via both routes. Both tactile and transilluminating impressions seen by the opposing endoscope allow for precise and safe passage of the guidewire through the obstruction. In addition, multiplanar fluoroscopic guidance helps determine the length of the obstruction and alignment of the dual endoscope. Guidewires, however, are not the only devices used to traverse the obstruction. Good success also has been documented with the needle knife [13,22], biopsy forceps [19] , cup forceps [17], EUS-N $[13,18]$ and balloon dilators $[15,19]$. Once luminal patency is achieved, a guidewire is usually passed through the mouth or G-tube site followed by balloon dilation over the wire. A nasogastric tube can be inserted to ensure access to the stricture for the next dilation within 24-48 hours. The nasogastric tube is then removed and further serial dilations resumed, as needed, over subsequent few weeks. In the past, to maintain luminal patency over the following few months, some investigators have passed a loop of string through the nostril, which traverses the neo-esophagus, exits the G-tube tract, and is taped to the patient's abdomen. That, however, is cumbersome and associated with some patient discomfort because the string through the nose is present for a few months. As an alternative, when technically feasible, we have used a fully covered esophageal stent to maintain luminal patency after creation of the neoesophagus.

A limitation of the standard dual endoscope antegrade and retrograde endoscopy procedure that has been well documented has been its poor procedural success in obstructions longer than 3 $\mathrm{cm}[12,16,22]$. The crux of this issue lies in the feasibility of having two endoscopes approach each other and align in close proximity, as determined by multiplanar fluoroscopy and visible transillumination. Until recently, when patients were determined not to be candidates for endoscopic lumen restoration based on longer lengths of obstructed esophagus, they were referred for surgery, which often is not feasible in individuals with multiple comorbidities and a prior history of surgery and irradiation to this area. However, we have recently circumvented this problem by describing a unique approach, POETRE, which borrows from an application used during peroral endoscopic myotomy (POEM). POETRE and similar techniques have been described in the literature in limited case reports $[8,26,27]$ with good success. With POETRE, a neo-esophagus can be created through submucosal tunneling into obstructions previously felt to be too long for standard rendezvous procedures.

Postoperative care usually is dependent upon the type of procedure performed. Typically, patients undergoing procedures other than POETRE were discharged or admitted for overnight observation with slow progression of their diet. Repeat endoscopic dilation within 24 to 48 hours was performed to ensure there was no restenosis. Post-procedural imaging or antibiotics were usually not necessary in the absence of symptoms. After POETRE, periprocedural antibiotics were given at our center for at least 5
Table 4 Published adverse events after endoscopic therapy for complete esophageal obstruction

\begin{tabular}{|l|l|}
\hline Adverse events & Patients \\
& $(\mathbf{n = 3 6 )}$ \\
\hline (Micro) perforations, $\mathrm{n}(\%)$ & $13(36)$ \\
\hline Mediastinal emphysema [12, 18] & 2 \\
Subcutaneous emphysema [12] & 1 \\
\hline Both [17, 20, 22] & 10 \\
\hline G-tube malfunction, $\mathrm{n}(\%)[17,19]$ & $9(25)$ \\
\hline Pneumothorax, $\mathrm{n}(\%)[15,17,22]$ & $4(11)$ \\
\hline Infection, $\mathrm{n}(\%)$ & $4(11)$ \\
\hline Paraspinal abscess [20] & 1 \\
\hline Periesophageal abscess [18] & 1 \\
\hline Cervical abscess [18] & 1 \\
Pharyngeal infection [17] & 1 \\
\hline Tracheoesophageal fistula, $\mathrm{n}(\%)[20]$ & $1(3)$ \\
\hline Esophageal perforation, $\mathrm{n}(\%)[16,17,22]$ & $3(8)$ \\
\hline Tooth avulsion, $\mathrm{n}$ (\%) [19] & $1(3)$ \\
\hline Death, $\mathrm{n}$ (\%) [19] & $1(3)$ \\
\hline
\end{tabular}

days. Patients were typically started on a liquid diet the following day and discharged within 48 to 72 hours.

Nearly all of the procedures described for CEO have had a good technical success rate and clinical outcome. However, a limitation of such studies is the uncontrolled nature of how cases were described. Standardization in reported results can aid in circumventing this issue. For instance, one objective measure that can be used is reporting a dysphagia score $[8,19]$. Such a score provides an objective measurement of outcome and is routinely used at our center and in this study as well. Clinical success was typically displayed in most reports, with a cumulative rate of $78 \%$ (range $0 \%-100 \%$ ). This wide range of outcomes can be explained by the lack of homogenous patient populations, subjective reporting, and diversity in intra-procedural technical approaches. Furthermore, it is important to note that influences on clinical success are not inherently dependent on restoring luminal patency. Previously sustained damage of muscles and nerves involved in deglutition from radiation are a likely explanation for lack of symptomatic improvement despite successful reconnection of the obstructed esophagus [28]. Indeed, studies have shown that establishment of esophageal continuity is not the only requirement for regaining the swallowing function; it is likely a multifactorial process. To that end, more recent studies have aimed to determine predictors of functional outcome versus just technical success [22]. At our institution, as at other centers that have previously published series, we did not encounter any procedural AEs. However, as mentioned earlier, accurate reporting of AEs is likely best achieved in prospective studies using objective predetermined criteria as suggested by the ASGE [10].

\section{Conclusions}

Combined antegrade and retrograde endoscopic therapy appears to be a safe and efficacious minimally invasive option for treatment of CEO. Various endoscopic techniques and novel accessories have been used via this route to reconnect the obstructed esophagus, including our recent description of POETRE using submucosal tunneling.

\section{Competing interests: None}




\section{Reference}

1 Lawson JD, Otto K, Grist W et al. Frequency of esophageal stenosis after simultaneous modulated accelerated radiation therapy and chemotherapy for head and neck cancer. Am J Otolaryngol 2008; 29: 13 - 19

2 Hernandez LV, Jacobson JW, Harris MS. Comparison among the perforation rates of Maloney, balloon, and savary dilation of esophageal strictures. Gastrointest Endosc 2000; 51: 460-462

3 Zhou JH, Jiang YG, Wang RW et al. Management of corrosive esophageal burns in 149 cases. J Thorac Cardiovasc Surg 2005; 130: 449-455

4 Tucker GF. Cicatricial stenosis of the esophagus with particular reference to treatment by continuous string retrograde bougienage with the author's bougie. Ann Otol Rhinol Laryngol 1924; 3: 1180-1213

5 van Twisk JJ, Brummer RJ, Manni JJ. Retrograde approach to pharyngoesophageal obstruction. Gastrointest Endosc 1998; 48: 296-299

6 De Barbosa JC, Da Rocha RM, Da Cunha FC. Impenetrable cicatricial obliteration of the thoracic esophagus; treatment by combined thoracotomy and endoscopy, with report of two cases. J Am Med Assoc 1953; 152: $1103-1105$

7 Bueno R, Swanson SJ, Jaklitsch MT et al. Combined antegrade and retrograde dilation: a new endoscopic technique in the management of complex esophageal obstruction. Gastrointest Endosc 2001; 54: 368 372

8 Wagh MS, Yang D, Chavalitdhamrong D et al. Per-oral endoscopic tunneling for restoration of the esophagus (POETRE). Gastrointest Endosc 2014; 80: 330

9 Vakil N, Morris AI, Marcon $N$ et al. A prospective, randomized, controlled trial of covered expandable metal stents in the palliation of malignant esophageal obstruction at the gastroesophageal junction. Am J Gastroenterol 2001; 96: 1791 - 1796

10 Cotton PB, Eisen GM, Aabakken $L$ et al. A lexicon for endoscopic adverse events: report of an ASGE workshop. Gastrointest Endosc 2010; 71: $446-454$

11 Lew RJ, Shah JN, Chalian A et al. Technique of endoscopic retrograde puncture and dilatation of total esophageal stenosis in patients with radiation-induced strictures. Head Neck 2004; 26: 179-183

12 Maple JT, Petersen BT, Baron TH et al. Endoscopic management of radiation-induced complete upper esophageal obstruction with an antegrade-retrograde rendezvous technique. Gastrointest Endosc 2006; 64: $822-828$

13 Moyer MT, Stack BCJr, Mathew A. Successful recovery of esophageal patency in 2 patients with complete obstruction by using combined antegrade retrograde dilation procedure, needle knife, and EUS needle. Gastrointest Endosc 2006; 64: 789-792

14 Garcia A, Flores RM, Schattner $M$ et al. Endoscopic retrograde dilation of completely occlusive esophageal strictures. Ann Thorac Surg 2006; 82: $1240-1243$
15 Steele NP, Tokayer A, Smith RV. Retrograde endoscopic balloon dilation of chemotherapy- and radiation-induced esophageal stenosis under direct visualization. Am J Otolaryngol 2007; 28: 98-102

16 Dellon ES, Cullen NR, Madanick RD et al. Outcomes of a combined antegrade and retrograde approach for dilatation of radiation-induced esophageal strictures (with video). Gastrointest Endosc 2010; 71: $1122-1129$

17 Goguen LA, Norris CM, Jaklitsch MT et al. Combined antegrade and retrograde esophageal dilation for head and neck cancer-related complete esophageal stenosis. Laryngoscope 2010; 120: 261 - 266

18 Schembre D, Dever JB, Glenn $M$ et al. Esophageal reconstitution by simultaneous antegrade/retrograde endoscopy: re-establishing patency of the completely obstructed esophagus. Endoscopy 2011; 43: 434437

19 Fowlkes J, Zald PB, Andersen P. Management of complete esophageal stricture after treatment of head and neck cancer using combined anterograde retrograde esophageal dilation. Head Neck 2012; 34: $821-825$

20 Boyce HW, Estores DS, Gaziano J et al. Endoscopic lumen restoration for obstructive aphagia: outcomes of a 25 -year experience. Gastrointest Endosc 2012; 76: 25 -31

21 Gavriel H, Duong C, Spillane J et al. Bidirectional esophageal dilatation in pharyngoesophageal stenosis postradiotherapy. Head Neck 2013; 35: $733-737$

22 Grooteman KV, Wong Kee Song LM, Vleggaar FP et al. Functional outcome of patients treated for radiation-induced complete esophageal obstruction after successful endoscopic recanalization (with video). Gastrointest Endosc 2014; 80: 175-181

23 Zald PB, Andersen PE. Fatal central venous air embolism: a rare complication of esophageal dilation by rendezvous. Head Neck 2011; 33: $441-444$

24 Eisdorfer RM, DiLorenzo JC, Miskovitz P. A complication of PEG change. Gastrointest Endosc 1991; 37: 108

25 Gornals JB, Consiglieri C, Castellvi JM et al. Treatment of complete esophageal stenosis using endoscopic ultrasound-guided puncture: a novel technique for access to the distal lumen. Endoscopy 2014; 46: 01 UCTN E2 - 3

26 Kachaamy T, Lott D, Crujido LR et al. Esophageal luminal restoration for a patient with a long lye-induced stricture via tunnel endoscopic therapy during a rendezvous procedure followed by self-dilation (with video). Gastrointest Endosc 2014; 80: 192 - 194

27 Babich JP, Diehl DL, Entrup MH. Retrograde submucosal tunneling technique for management of complete esophageal obstruction. Surg Laparosc Endosc Percutan Tech 2012; 22: e232 - 235

28 Coia LR, Myerson RJ, Tepper JE. Late effects of radiation therapy on the gastrointestinal tract. Int J Radiat Oncol Biol Phys 1995; 31: 1213 1236 